\title{
Donor cell derived deletion $7 q$ occurring after allogeneic stem cell transplantation: a case report
}

\author{
Anke Delie ${ }^{1}$, Philip Vlummens ${ }^{1}$, Nadine Van Roy², Fritz Offner ${ }^{1}$, Tessa Kerre ${ }^{1}$
}

1Department of Hematology, Ghent University Hospital, Belgium

${ }^{2}$ Centre for Medical Genetics Ghent, Ghent University, Belgium

\section{INTRODUCTION}

Deletion $7 q(\operatorname{del}(7 q))$ is a non-reciprocal chromosomal abnormality which appears to play a significant role in the development and progression of myeloid malignancies (myelodysplastic syndrome (MDS), acute myeloid leukemia (AML) and juvenile myelomonocytic leukemia). It is frequently seen in patients with therapy related myeloid neoplasms and is associated with poor prognosis $(1,3)$. The resulting compound haploinsufficiency of multiple tumor suppressor genes and dysregulation of gene expression is thought to play a key role in disease development and progression $(1,2)$. Deletion $7 q$ has been found to be a recurrent genetic abnormality in donor cell leukemia (DCL) and -MDS with an incidence of up to $36.4 \%$ (4).

\section{CASE PRESENTATION}

CLINICAL HISTORY \& DiAgNOSIS

Our patient initially presented with a diagnosis of poor risk AML (FLT3+/NMP1-) at the age of 56. Cytogenetic analysis at diagnosis showed a $3 p$ deletion and translocation $t(5 ; 11)$.

Four years earlier, the patient had been treated for a follicular lymphoma.

\section{AML TREATMENT}

Standard induction chemotherapy was initiated (Cytarabine and Idarubicine), followed by consolidation with Mitoxantrone and Etoposide. After consolidation he was referred for alloHSCT, with his HLA-identical sister acting as donor. Pre-transplant bone marrow aspirate showed a complete morphological and molecular remission without any cytogenetic abnormalities. On pre-donation screening, peripheral blood counts of the donor showed no abnormalities. The conditioning regimen consisted of Fludarabine, oral Busulfan and antithymocyte-globulin (ATG). Graft-versus-host-disease (GVHD) prophylaxis consisted of tacrolimus and mycophenolate mofetil, in adition to the ATG as part of the conditioning. A total of $2,15 \times 10 \mathrm{E} 6$ CD34+ peripheral blood stem cells/kg was administered.

POST-TRANSPLANT COURSE

- Transient clonal expansion of T-cell large granular lymphocytes (TLGL) at 2 years post-transplant, present in bone-marrow aspirate and peripheral blood. A spontaneous resolution was seen after 2 years.

- Routine bone marrow analysis 4 years post-transplant identified a $\operatorname{del}(7 q)$ in 10 out of 20 cells, all of them with female karyotype $(46, X X, \operatorname{del}(7)(q 21)[10])$, alongside normal morphology and full donor chimerism. Retrospective analysis of a previous bone marrow sample showed a del(7q) in 1 out of 20 cells, indicating progression of the clone. The donor was contacted and bone marrow analysis showed no morphological or cytogenetic abnormalities.

\section{KARYOTYPE}

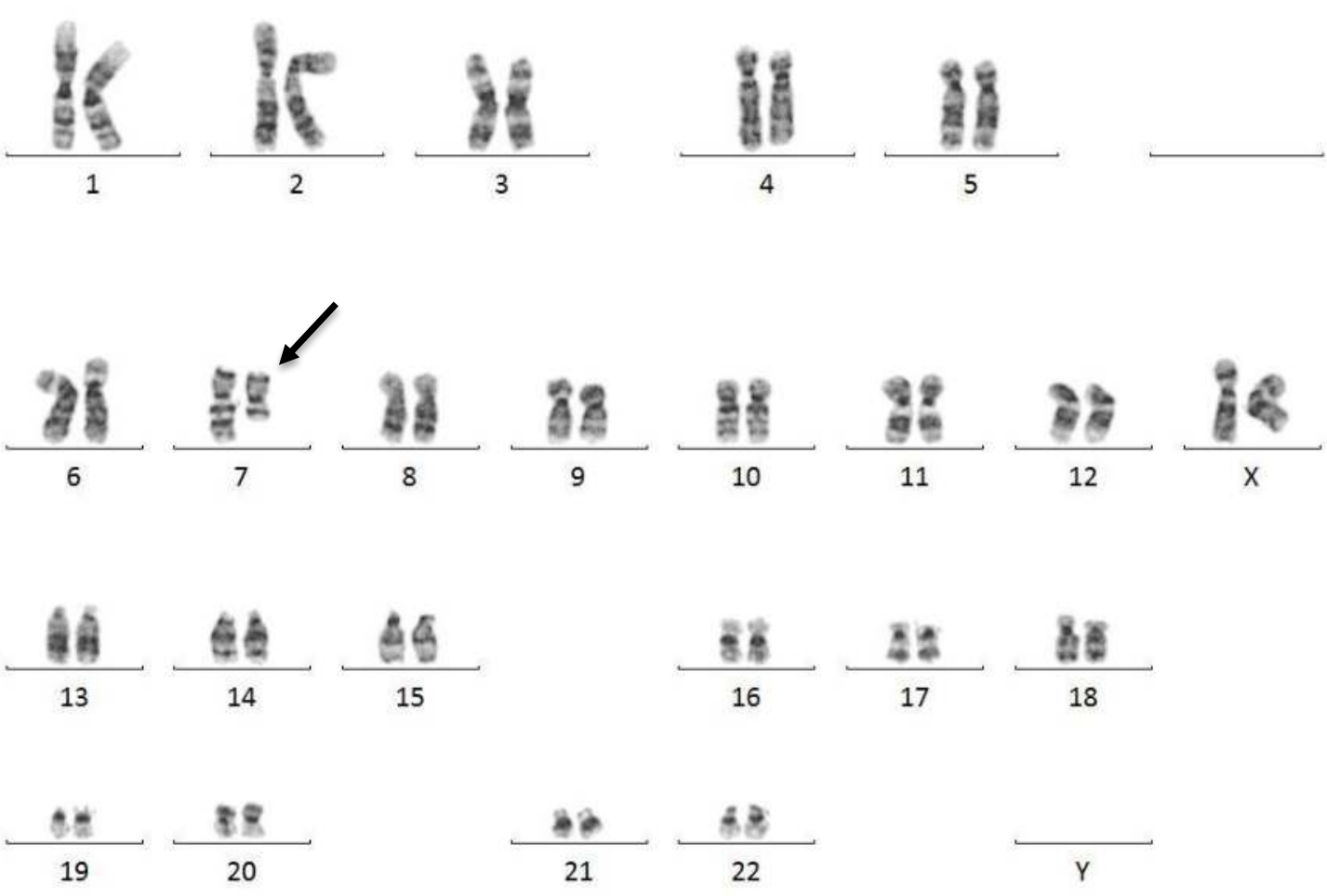

\section{DONOR CELL LEUKEMIA}

DCL occurs when de novo acute myeloid leukemia occurs in engrafted cells of donor origin, which can be found in up to $5 \%$ of all relapses post alloHSCT. The pathogenesis is however poorly understood. Possible mechanisms include (5):

1. Abnormal hematopoietic micro-environment, pre-existing or induced by delayed effects of cytotoxic treatment.

2. Defective immune surveillance, either as a predisposing condition or induced post-transplant by impaired immune reconstitution or immunosuppressive treatment.

3. Prior genetic susceptibility to disease existing within donor cells. 4. Telomere shortening due to proliferative demand after HSCT.

5. Fusion of leukemogenic DNA released from leukemia cells by conditioning with donor cell DNA or fusion of residual leukemia cells with donor stem cells.

\section{DISCUSSION}

The mechanism of development of $\operatorname{del}(7 q)$ in our patient is unclear. However, when reviewing our case it seems most likely that a preexisting host factor such as an impaired bone marrow microenvironment or defective immune surveillance played a significant role in its development in our patient. The past history of a low grade lymphoproliferative disorder, the occurrence of a T-LGL post-transplant and the long latency between transplant and development of the abnormality all support this theory.

There is a high risk of further clonal evolution either to donor cell derived MDS or DCL underlining the need for strict further follow-up.

- The routine use of cytogenetic analysis in patients post alloHSCT could lead to the early detection of de novo clonal abnormalities with an impact on survival.

- Detection of donor cell derived clonal abnormalities post alloHSCT should warrant a thorough evaluation of the donor if possible as well as a strict follow-up of the patient for clonal evolution to DCL or donor cell MDS.

- Donor cell leukemia is a rare finding but must be considered in all post alloHSCT leukemia or MDS cases.

1. Honda H, Nagamachi A, Inaba T. -7/7q- syndrome in myeloid-lineage hematopoietic malignancies: attempts to understand this complex disease entity. Oncogene. 2015;34(19):2413-25.

2.Inoue K, Fry EA. Haploinsufficient tumor suppressor genes. Adv Med Biol. 2017;118:83-122.

3.Pellagatti A, Boultwood J. The molecular pathogenesis of the myelodysplastic syndromes. Eur J Haematol. 2015;95(1):3-15

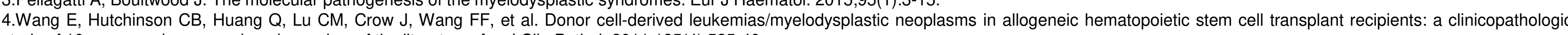

